\title{
nature physics
}

It's World Year of Physics - what better time to launch a physics journal?

But is there really a need for another journal? We believe, wholeheartedly, that the answer is yes. Research in physics, whether pure or applied, is as vibrant as ever. There are many journals in which to publish. There are magazines too, offering more informal material, a chance to take a wider view of a burgeoning field. But never before has there been a physics publication that is both, journal and magazine. Nature Physics is it.

Nature Physics springs from the Nature fold. It shares the philosophy of its parent title, in presenting highquality primary research complemented by a range of commissioned and editorial material. Each issue has at its core a collection of Letters and Articles, recounting original research that is novel, significant and of interest to a broad audience of physicists. Some of those papers, and others from the wider literature, are discussed in our News and Views section - each expert-written account putting the development into context for the (perhaps less expert) reader. Among the other sections, Commentary offers the opinions of physicists and others on the issues that exercise them, be they policy or politics, or even physics; Thesis features our columnists Mark Buchanan and Lawrence Krauss; and Endgame, well, that's just for fun.

Our first foray into extending the physics coverage of Nature came in 2001 with the launch of the Nature Physics Portal. The research highlights, plus a round-up of physics-relevant content from Nature, offered on that site proved to be a winning formula, drawing tens of thousands of visitors each week. In many ways, not least for the response it enjoyed, the portal paved the way for Nature Physics, the journal.

The efficacy of the web for reaching as many readers as possible, as quickly as possible, underpins the practice of our new journal. All research articles in Nature Physics are published online first, ahead of the print issue (what we call 'Advance Online Publication'). Those pressed for time (who isn't?) may like to register on our website for an electronic 'table of contents' alert - a monthly bulletin, delivered straight to your e-mail inbox, with highlights from the latest issue. For those whose personal reading time is (like ours) mostly snatched on planes or trains or in the bath, Nature Physics is also available by subscription in good old-fashioned print.

Authors may be pleased to know that manuscripts can be submitted to Nature Physics not only in Microsoft Word, but in LaTeX too. This seemed to us an essential criterion for the launch of a physics title, as these wordprocessing packages are so widely used in the physics community. What every physicist loves about LaTeX is the ease with which equations can be formatted and, subsequently, the elegance of their appearance on the page. Among the Letters and Articles in each of our issues, you will find some very elegant equations, benefitting from a typesetting process that happens entirely in LaTeX.

Something that isn't a new development, but a policy shared by all Nature titles, is that we do not object to papers being posted on preprint servers. We would only advise that, if you do so ahead of publication of your manuscript in Nature Physics, you risk diluting the impact of your paper in the media and the wider scientific community. But preprint servers - arXiv.org in particular - are integral to the working life of physicists. Far from banning any posting, we applaud the communication that the servers foster among researchers.

And what of the Physics Portal? That site is evolving in response to the development of physics publishing within the Nature Publishing Group, whose portfolio now includes Nature Physics and Nature Materials alongside Nature itself. To reflect this expansion of interests in the physical sciences, the Portal - in its new guise as physics@nature.com - will now serve to highlight physics-relevant material published across all of these titles.

'Physics' is huge remit, but one that we are tackling with relish. We hope that every physicist feels that their interests are represented here - in the reviews, commentaries, News and Views, as well as the primary papers. We also hope that you will enjoy this opportunity to explore physics more widely, whether for the purposes of your own research, or purely for pleasure.
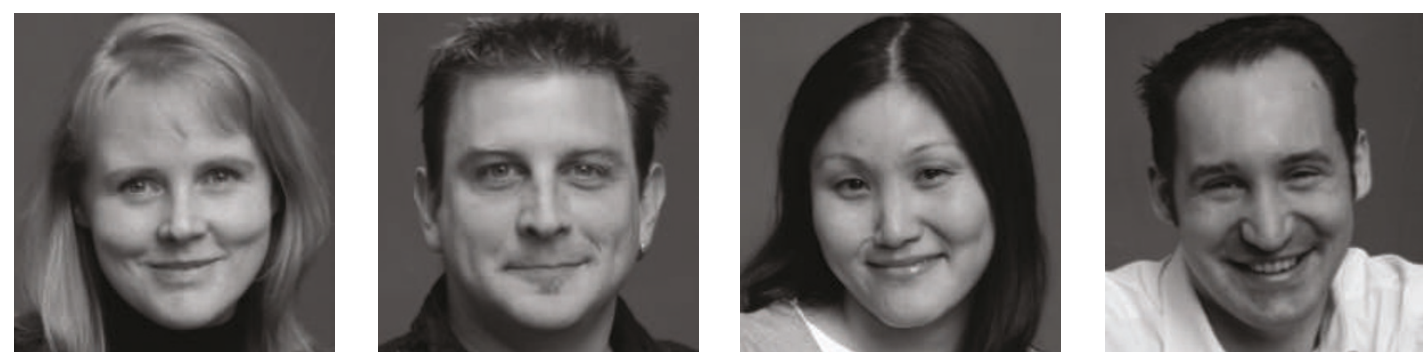